\title{
THE KINETIC THEORY OF OSMOTIC PRESSURE.
}

\author{
Professor Alfred W. Porter, D.Sc., F.R.S., presented the following \\ Paper on "The Kinetic Theory of Osmotic Pressure" :-
}

It is not my intention to summarize the present state of our knowlectge in regard to osmotic pressure. It is not many years since a discussion on the subject took place in this Society (January 29, 1907), in the course of which some account was given of the state of experimental knowledge at that time and of theoretical explanations of the experimental facts. Since that time there has been a considerable amount of work done both on the experimental and the theoretical side, and this must claim our attention. Quite a large part of this is free from any disputatious matter; especially the part which rests upon thermodynamical relations. On the other hand, when we turn to the causes of osmotic pressure, no unanimity is yet to be found, and on this point I have some remarks to make. Again, no full examination has yet been made of Morse and Frazer's experimental results ; these, also, claim one's attention.

As the term "osmotic pressure" does not possess an invariable meaning, it is necessary to state that $\mathbf{I}$ shall always mean by it the equilibrium difference of pressure between a solution and the pure solvent when separated by a membrane permeable to the solvent alone. In any solution the masses of solvent and solute will be denoted by $\mathbf{M}$ and $m$ and the corresponding numbers of molecules by $\mathrm{N}$ and $n$.

\section{The Kinetic Theory of Osmotic Pressure.}

I consider first the kinetic theory of osmotic pressure. It is better to speak of kinctic theory than gas theory, because the latter term is often taken to imply conformity with the laws of perfect gases. No upholder of the theory has ever supposed that the perfect gas laws for osmotic pressure would hold good in all cases; but that they would be approximated to in the case of dilute solutions only. On the other hand, opponents of the theory have made the most of the fact that strong solutions exhibit great deviations from those laws. These deviations, of course, no more militate against the kinetic theory than do analogous deviations in the case of gases themselves. The greatest triumphs of the kinetic theory have been obtained (by Hirn, van der Waals, Dieterici, and others) in, at least partially, accounting for the complexities in the behaviour of gases. Yet.similar complications in the case of osmosis are frequently supposed to be fatal to the theory. In spite of the fact that this theory is the only one which directly gives the experimentally obtained values for dilute solutions, it has met with great opposition both from physicists and from certain schools of chemists. The strength on the side of the opponents lies in a recognition of the fact that the conditions in a liquid are certainly not identical in every respect with those in which the molecules of a gas 


\section{I24 THE KINETIC THEORY OF OSMOTIC PRESSURE}

move. However, our knowledge in regard to liquids has increased extensively in the last few years. There is now an experimental basis for a kinetic theory of liquids supplied by the investigations of Perrin and others on Brownian motion. Particles suspended in a liquid are in rapid motion to the precise amount required by theory. This statement must apply to the molecules of a solute also ; and the dynamical effect of this motion must needs be taken into account. This effect, calculated according to legitimate methods, comes out equal to the experimental value of osmotic pressure. Any other theory put forward to account for osmosis must fulfil, then, a double duty : not only must it be competent to explain osmosis, but it must also explain away the effects that we have the right to expect from the molecular agitation of the solute.

The curious objection is often raised that the pressure according to the kinetic theory of gases goes the wrong way. This objection would hardly deserve notice if it were not for its extreme vitality. A controversy has recently taken place between van Laar and Ehrenfest* on this point; it is reported in Nature for March 16, I9I6.

In van Laar's opinion the pressure of the sugar molecules against the semipermeable membranes would drive the water out.

The consideration of the subject is usually complicated unnecessarily by endeavouring to deal at the outset with real molecules occupying space and attracting and attracted by all other molecules present. Warning should be taken from the difficulties which arise in the kinetic theory of gases when this thoroughgoing procedure is adopted. It is better to suppose the solute to consist of moving massive points ; for then its introduction into a solvent does not displace the molecules of the latter. Much of the difficulty is thereby removed.

Consider a long vessel of water under uniform pressure throughout. In the left hand half disperse a number of ideal sugar molecules so as to form a dilute solution. If left alone they will slowly diffuse throughout the water. The rate of diffusion will, of course, depend upon the viscosity of the water ; but this rate does not concern us. In order to prevent diffusion a force from right to left must be introduced, acting only upon the sugar. It does not matter how this force is introduced. A row of demons, clinging to fixed supports, would serve-each with instructions to keep the sugar molecules from getting through. These demons require to exert no force so far as the water is concerned, because it presses upon them equally on all sides.

The pressure of the sugar arises from its thermal motion; and Perrin has given evidence that this is the same as for a gas. The reversal of this motion at the screen requires the same pressure as for the corresponding case of a gas. This is the osmotic pressure. The sides of the vessel (or any body immersed in the solution) experience pressure due both to sugar and water; and therefore equilibrium exists with a difference of pressure between the two sides of the semi-permeable screen, the balance being taken up by the screen.

In order to obtain a picture of the principal effect of the presence of a solute we may regard it as helping, by its molecular bombardment, to enlarge the boundaries of the solution, and thereby to relieve the total pressure on the solvent. If the solution be now put into communication with the solvent at the same external pressure, the latter flows in. This process has recently been called osmotic suction, and perhaps this name describes it well enough, though I doubt if "suction" is a scientific word.

- Ehrenfest, Proc. Amsterdam Acad., xvii. pp. I24I-45 ; J. J. van Laar, ibid., xviii. pp, 184-90. 
But it must be remembered that, however interesting the processes concerned with the flow of the solvent may be, the quantity which we call the osmotic pressure is concerned primarily with the equilibrium state; it is an outwardly directed pressure upon the semi-permeable membrane, and it is given in amount by the bombardment pressure of the solute against that membrane-at least in the case of dilute solutions.

Of course, when the solvent and solute are both gaseous the modus operandi (as sketched above) is, I believe, admitted by all. Hot silica is permeable to hydrogen, but not to nitrogen ; and it constitutes, therefore, a selective membrane. Let a cylinder, with piston, have such a membrane fitted to it; and, when containing nitrogen, immerse it in hydrogen. Hydrogen enters, and the vessel expands, unless additional pressure is exerted upon the piston. In this case the total pressure that will keep the volume unchanged is the sum of the pressures of the two gases. Attractive forces, surface tension, etc., have nothing to say to it, because these forces do not exist.

These statements, applied to liquids, require that the solution shall be very dilute. In such cases the aggregates, of which the molecules of the solute arc the centres, are so far apart from one another that further dilution of the solution will merely separate them without interfering with the constitution of any of them. The change of mechanical energy which arises from further dilution is then independent of the kind of matter involved, and will depend only upon the number of such aggregates and be the same for all systems which agree in this respect. "One such system is an ideal gas, there being now no solvent; it follows that the osmotic pressure of the molecules of the dissolved substance against an internal partition, permeable to the solvent and not to them, is the same as if they existed in the state of an ideal gas at their actual density and temperature." *

This equality can be proved also by means of a thermodynamical cycle, such as that made use of by Lord Rayleigh ; $\nmid$ but, of course, any such proof gives no hint of the mechanism by which the equality is brought about.

It will be seen that I find it impossible to put myself in the mental position of FitzGerald in his Helmholtz Lecture $\ddagger$ (which is one of the most noteworthy contributions to the cult of caution in the advocacy of scientific theories). He says: "It is no doubt a most remarkable thing that osmotic pressure should be even roughly the same as what would be produced by the molecules of the body in solution as if in the gaseous state; but to imply that the dynamical theory of the two is at all the same, or that the dynamical theory of a gas is in any sense an explanation of this law of osmotic pressure is not at all in accordance with what is generally meant by the word 'explanation." I wish to dissociate myself completely from the position adopted in this sentence.

Of course, when the processes by which the final equilibrium state are set up or the properties of the membrane which make it semi-permeable are examined, many interesting connections will certainly be found which will make it possible to express osmotic pressure in terms of them. It is often difficult to distinguish between cause and effect in the case of concomitant events. The kinetic pressure of the solute acts outwardly and diminishes the Laplacian pressure by an equivalent amount, and therefore connections may be found between osmotic pressure and Laplacian pressure. But the prime-mover, or causa causans, of the whole disturbance seems indubitably

\footnotetext{
- Larmor, Ether and Matter, p. 287.

+ Nature, lv. p. 253 (1897).

† Trans. Chem. Soc., I8g6.
} 


\section{I26 THE KINETIC THEORY OF OSMOTIC PRESSURE}

to be the molecular agitation of the solute, and a knowledge of this provides us with the only reay in which the value of the pressure has been calculated from theory.

In concentrated solutions it is unwarrantable to neglect the attractive forces and volume of the solute molecules; and indeed they may become the most important factor. To recognize this is in no way to give up the gas theory; unless, indeed, it is claimed that van der Waals gave up the theory for gases themselves when he wrote his famous treatise on the continuity of the liquid and gaseous states. Nor must it be expected that the equation for osmotic pressure will be identical with the characteristic equation of a simple gas. In the osmotic problem we are dealing with, at least, a binary mixture. This is a type of problem which has been tackled by the Dutch school of physicists ; but it is not surprising that their efforts at present are only tentative. The true characteristic equation for a simple fluid is not yet known; the problem of the binary mixture is a much harder one.

\section{The Semi-permeable Membrane.}

The properties of the membrane form an interesting and important but quite subsidiary problem. Provided it is truly semi-permeable, the value of the difference of pressure between its two sides is determined by the properties of the solution and solvent and not by those of the membrane. Its semi-permeability, no doubt, must be expressible in terms of its own properties. It has to exert a pressure which is equal to the aggregate of the forces required to stop the solute while affording a passage for the solvent. In order to act as such a membrane, any canals through which fluid can permeate must be narrow compared with the range of molecular forces. So long as this is the case there seems to be nothing to prevent them being so narrow that the passage of the solvent through them can be described as a process of solution in the material of the membrane ; or at least of that coarser kind of solution which is distinguished as colloidal. But it does not appear true that semi-permeability always arises in this way. The most perfectly semi-permeable membrane known (as FitzGerald pointed out) is the surface of separation between a solution and its vapour. Here, the sorting is effected in the Laplacian layer, which exerts such attraction on the sugar that it practically never succeeds in escaping through. Where solubility is the determining factor a study of the membrane will bring out the influence of the properties concerned with a variation of solubility and may throw light upon the nature of solutions. These, however, are collateral studies and have no more connection with osmotic pressure than the study of the elastic properties of a glass vessel has upon the origin of the pressure it experiences from the water contained in it.

\section{An Interpretation of Experimental Results.}

Experimental advance since 1907 has been made mainly by Morse and his collaborators in America and by the Earl of Berkeley and his co-workers in Britain. To Lord Berkeley we owe observations extending over the widest range of concentrations at one temperature only; to Morse we are indebted for a set extending over a fair range of concentrations but at various temperatures between $0^{\circ} \mathrm{C}$. and $80^{\circ} \mathrm{C}$. Lord Berkeley's values for cane sugar are given in Table $I$, together with values calculated according to the perfect gas law and Morse's values at $o^{\circ}$ for comparison. 
Cane Sugar. Temp. $\circ^{\circ} \mathrm{C}$.

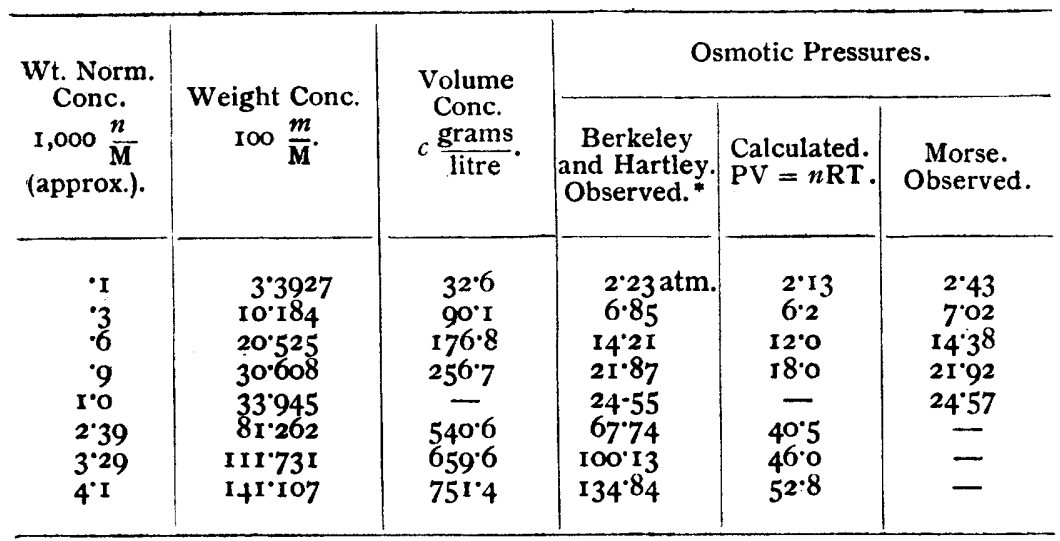

It will be seen that there is some disagreement between the two experimentalists; the discrepancy has not yet been explained. Both sets are in excess of the perfect-gas pressures even for the most dilute solutions. $\dagger$

Morse's values have the most importance for us, because they cover a wide range of temperature. They are given in the Table $\ddagger$ :-

Osmotic Pressure of Cane Sugar Solutions.

Weight-normal Concentration.

$\begin{array}{llllllllll}0.1 & 0.2 & 0.3 & 0.4 & 0.5 & 0.6 & 0.7 & 0.8 & 0.9 & 1 \cdot 0\end{array}$

\section{Mean Osmotic Pressure in Aimospheres.}

Temp.

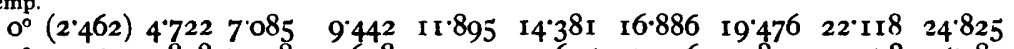

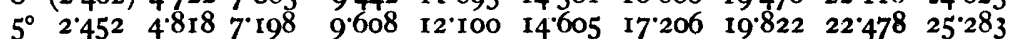

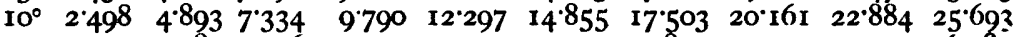

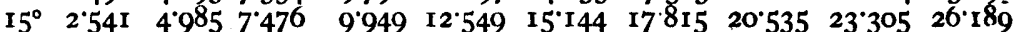

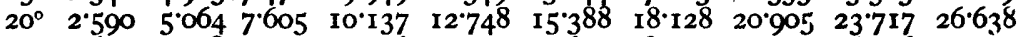

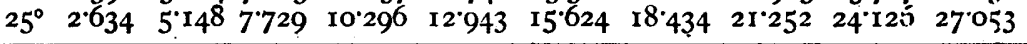

$\begin{array}{lllllllllll}30^{\circ} & 2.474 & 5.044 & 7.647 & 10.295 & 12 \cdot 978 & 15 \cdot 713 & 18.499 & 21 \cdot 375 & 24.226 & 27.223\end{array}$

$\begin{array}{lllllllllll}40^{\circ} & 2 \cdot 560 & 5 \cdot 163 & 7 \cdot 844 & 10 \cdot 599 & 13.355 & 16 \cdot 146 & 18 \cdot 932 & 21 \cdot 803 & 24 \cdot 735 & 27 \cdot 701\end{array}$

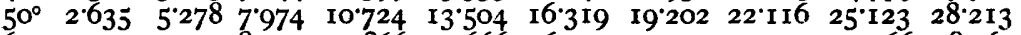

$\begin{array}{llllllllllll}60^{\circ} & 2 \cdot 717 & 5.437 & 8 \cdot 140 & 10 \cdot 866 & \text { I3 } 666 & 16 \cdot 535 & 19 \cdot 404 & 22 \cdot 327 & 25^{\circ} \cdot 266 & 28 \cdot 367\end{array}$

$70^{\circ}$ - - - - 13991 16.820 19.568 22.567 25.562 28.624

$80^{\circ}$ - — - -

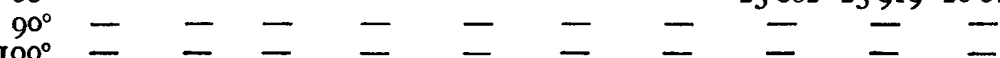

* Berkeley and Hartley, Proc. Roy. Soc., A, 92, p. 483 (I9I6).

$\dagger$ A suggested explanation of this has been given by G. N. Lewis, $\mathscr{F}$. of Amer. Chem. Soc., vol. xxx., No. 5, May 1908.

$\ddagger$ Morse, Holland, Myers, Cash, and Zinn, Amer. Chem. F., 48, 29 (1912).

$\$$ The last value in the table is given as 28.000 by Morse and also (by quotation) by other writers. A comparison with Morse's detailed table shows that it should be 28.818 . 


\section{I28 THE KINETIC THEORY OF OSMOTIC PRESSURE}

Morse exhibits the deviations from a fictitious gas law by calculating the ratios of these numbers to the values of the pressures of a gas containing the same numbers of molecules in a volume equal to the volume (not of the solution but) of the pure solvent at $4^{\circ} \mathrm{C}$. This process is so arbitrary that it leaves one with a very vague sense of what his ratios signify. I therefore do not quote them.

In a letter to Lord Berkeley about eight years ago I showed that his values could be calculated approximately from a formula of Hirn's type-

$$
\mathrm{P}(v-b)=\mathrm{RT}
$$

where $b$ is a constant not much different from the volume of sugar present. He did not think that the formula did complete justice to the accuracy of his experiments, and I am inclined to agree with him. To show the capability of this formula of giving a second approximation, I append a table in which it is applied to Morse's values at $20^{\circ} \mathrm{C}$. and in which $b$ is taken as 3 ro litres/mol.

\begin{tabular}{|c|c|c|c|}
\hline \multirow{2}{*}{$\begin{array}{c}\text { Weight Normal } \\
\text { Concentration. } \\
\qquad 1,000 \frac{n}{M}\end{array}$} & \multirow{2}{*}{$\begin{array}{l}\text { Volume Normal } \\
\text { Concentration. } \\
\text { grammol. } \\
c \frac{\text { litre }}{}\end{array}$} & \multicolumn{2}{|c|}{ Osmotic Pressure. } \\
\hline & & Observed. & Calculated.* \\
\hline $\begin{array}{r}\cdot \text { I } \\
\cdot 2 \\
\cdot 3 \\
\cdot 4 \\
\cdot 5 \\
\cdot 6 \\
7 \\
\cdot 8 \\
9 \\
1 \cdot 0\end{array}$ & $\begin{array}{r}\cdot 098 \\
\cdot 192 \\
\cdot 282 \\
\cdot 369 \\
\cdot 452 \\
533 \\
\cdot 510 \\
\cdot 584 \\
\cdot 756 \\
\cdot 825\end{array}$ & $\begin{array}{r}2 \cdot 59 \\
5 \cdot 06 \\
7 \cdot 61 \\
10 \cdot 14 \\
12 \cdot 75 \\
15 \cdot 39 \\
18 \cdot 13 \\
20.91 \\
23.72 \\
26.64\end{array}$ & $\begin{array}{r}2.43 \\
4.91 \\
7.43 \\
10.02 \\
12.64 \\
15.35 \\
18.09 \\
20.87 \\
23.74 \\
26.64\end{array}$ \\
\hline
\end{tabular}

The agreement at high concentrations is excellent; but is not so good for the more dilute solutions. The volume of a gram molecule of solid sugar is about $\cdot 2 \mathrm{I} 4$ litre. The high value 310 which is required might be accounted for by assuming that the sugar is hydrated in solution, the difference, 0.96 litre, representing the water of hydration. This would correspond to $96 / 18$ or 5.3 molecules of water associated with each molecule of sugar on the average. Callendar had previously found by a different line of argument the value 5 from Berkeley's numbers.

It is necessary, however, to examine all of Morse's numbers, and instead of assuming a constant value of $b$ for each temperature it is better to work backwards and find the value of $b$ for each concentration and temperature which would make the Hirn equation correct; and thence to determine the hydration number for each case. These are given on the opposite page.

Only the values up to $60^{\circ} \mathrm{C}$. are given, because no table is available of the densities of sugar solutions at higher temperatures; and these densities are needed in calculating the table.

* From formula $\mathrm{P}(\mathrm{I}-3 \mathrm{I}(\mathrm{c})=c \mathrm{R} 293$. 
HYDRATION NUMBERS.

\begin{tabular}{|c|c|c|c|c|c|c|c|}
\hline Wt. Cunc. & $0^{\circ}$. & $10^{\circ}$ & $20^{\circ}$ & $30^{\circ}$. & $40^{\circ}$ & $50^{\circ}$. & $60^{\circ}$ \\
\hline$\cdot$ I & 53 & 43 & 45 & 3 & 6.9 & $8 \cdot 8$ & II 5 \\
\hline$\cdot 2$ & I 5.8 & 16 & $\begin{array}{l}75 \\
16\end{array}$ & 7 & 5.5 & 45 & 58 \\
\hline 3 & IO' 3 & $10 \cdot 3$ & I I 2 & 6.7 & 60 & $4^{\prime} \mathrm{I}$ & 35 \\
\hline 4 & $7 \% 7$ & 8\% & $8 \cdot 3$ & 63 & $6 \cdot 4$ & 43 & $2 \cdot 8$ \\
\hline .5 & 69 & 6.8 & $7^{\circ} 2$ & 60 & 60 & 43 & $3^{\circ} 0$ \\
\hline .6 & 6.4 & $6 \cdot 3$ & 6.5 & 57 & 57 & 43 & $3^{2}$ \\
\hline 7 & 59 & $6 \circ$ & $6 \cdot 3$ & $5 \cdot 6$ & $5^{2}$ & 43 & $3^{2}$ \\
\hline .8 & 57 & 57 & 6.5 & 56 & $5^{\circ} \mathrm{O}$ & 42 & 33 \\
\hline 9 & $5 \cdot 5$ & 5.5 & 5.8 & 54 & $5^{\circ} 0$ & 44 & 33 \\
\hline ro & 5.5 & 5.5 & $5 \cdot 8$ & 54 & $4^{\circ} 9$ & 45 & $3^{6} 6$ \\
\hline
\end{tabular}

For comparison, the values calculated in the same way from Berkeley's determinations are appended :--

\begin{tabular}{|c|c|c|c|c|c|c|c|}
\hline $\begin{array}{l}\text { Wt. mol. conc. ... } \\
\text { Volume conc. grams per }\end{array}$ & $\cdot \mathbf{r}$ & 3 & $\cdot 6$ & 9 & $2 \cdot 39$ & 3.29 & $4^{\cdot r}$ \\
\hline litre $\quad \ldots \quad \ldots$. & $32 \cdot 6$ & $90^{\circ} \mathrm{I}$ & $\mathbf{1} 76 \cdot 8$ & $256 \cdot 7$ & $540 \cdot 6$ & 6596 & $75^{1} 4$ \\
\hline Hydration number $\quad \ldots$ & 14 & 17.6 & $8 \cdot 0$ & 53 & 48 & 44 & 40 \\
\hline $\begin{array}{c}\text { Mol. of water present per } \\
\text { mol. sugar }\end{array}$ & 550 & 183 & 82 & 61 & 24 & $16 \cdot 5$ & 134 \\
\hline
\end{tabular}

Both sets agree in giving diminishing hydration with increasing concentration. In other respects, the agreement is not too close; but it must be remembered that in dilute solutions in particular the degree of hydration has an exceedingly small infuence on the pressure.

The values obtained for dilute solutions at low temperatures seem surprisingly large; but I am not aware of any argument which makes them impossible. If we represent the volumes of sugar and water molecules by their representative spheres these will have radii in the ratio of 6 to 2.6 . Between 30 and 40 molecules of water would form a single layer on a sugar molecule. (The actual number would depend upon the nature of the packing.)

Now, it is exceedingly probable that the molecules of water immediately contiguous to each sugar molecule tend to form a condensed layer round it. No doubt this association, as in all other cases of molecular equilibrium, is only a kinetic one; these layers will continually be breaking up and re-forming. The hydration that can be found experimentally will represent an average or statistical value of the number of molecules in the condensed laver. Thus the values obtained from Berkeley's numbers come well within the range of probability. As the amount of water present per each molecule of sugar becomes less, the hydration becomes less, as we should expect it to do. In the strongest solutions used by Morse there are about 55 molecules of water present per molecule of sugar. Hence, in this case about one-tenth of the total amount forms the condensed or associated layer. In the strongest solutions employed by Berkeley there are 13.4 molecules of water present and 4 in the condensed layer--in each case per molecule of sugar.

I see nothing in these results to indicate the degrec of association of the water molecules with each other-mono-, di-, trihydrol are indistinguishable.

VOL. XIII-T5* 
Perhaps other contributors to this discussion will have evidence to bring forward bearing upon this side of the question.

Callendar in 1908 * came to the conclusion that the sugar molecules are hydrated in solution from a quite different line of reasoning. He modified a theory due to Poynting, + and assumed that the ratio of the saturation vapour pressures of solution and pure solvent $\left(\pi_{\pi} / \pi_{0}\right)$ is "equal to the ratio of the number of free molecules of solvent to the whole number of molecules in the solution, instead of to the number of molecules of solvent. If each molecule of solute appropriates $a$ molecules of solvent and if $n, \mathrm{~N}$ denote, as before, the whole number of molecules of solute and solvent respectively in the solution, the number of free molecules of solvent is $\mathrm{N}-$ an and the whole number of molecules in solution is $\mathrm{N}-a n+n$." He thus obtains

or

$$
\frac{\pi}{\pi_{\circ}}=\frac{\mathrm{N}-a n}{\mathrm{~N}-a n+n}
$$

$$
\frac{\pi_{\circ}-\pi}{\pi}=\frac{n}{\mathrm{~N}-a n} \text {. }
$$

According to the classical formula for dilute solutions $a=0$; according to Poynting's theory $a=\mathrm{I}$; Callendar tinds for cane sugar the value 5 from various physicochemical properties; but for high concentrations as determined from boiling-points near $100^{\circ} \mathrm{C}$. he finds it to diminish gradually to 3.

Apart from the some what arbitrary character of the above formula for the lowering of vapour pressure there is an essential difficulty in connection with it which requires further discussion. According to van't Hoff's original formula, obtained thermodynamically,

$$
\frac{\pi_{\mathrm{o}}-\pi}{\pi}=\frac{n}{\mathrm{~N}}
$$

$\mathrm{N}$ is explicitly the number of molecules of the solvent estimated of the same degree of complexity as in the vapour phase. In fact, it is a convenient abbreviation for $\mathrm{M} / \mu$, where $\mathrm{M}$ is the mass of solvent and $\mu$ is the molecular weight of water vapour. The number of real molecules of the solvent liquid does. not primarily enter into the problem at all. Now, water is believed to be associated, and therefore $\mathrm{N}$ is not the number of water molecules present even if hydration is neglected. I am puzzled therefore in endeavouring to understand in what sense $\mathrm{N}-a n+n$ is taken to be the total number of molecules present. This objection seems to strike at the very foundation both of Poynting's assumption and of Callendar's modification of it. There may be a way out of the difficulty, but the mode of escape has not yet been discussed or even hinted at.

On the totally different hypothesis which underlies the calculations of hydration in the present Paper, I have shown that the formula for the lowering can be written approximately

$$
\log \frac{\pi_{0}}{\pi}=\frac{n}{\mathrm{~N}-\mathrm{an}^{n}}
$$

in which $\mathrm{N}$ is explicitly the number of molecules of water reckoned as if normal and not the number of actual molecules present.

* Roy. Soc. Proc., A, vol. 8o, p. 482 (1908).

† Phil. Mag., vol. 42 , p. 298 (1896).

I It is noteworthy that, according to Poynting's theory, the osmotic pressure should be increased in the ratio $a$ when $a$ differs from unity. A discordance would arise in this connection; for no such increase is observed.

\$ Trans. Faraday Soc., vol. xi, part I, p. 27, 1915. 


\section{Latent Heats of Dilution.}

A test of the value of Morse's determinations can be made by aid of a knowledge of latent heats of dilution. I observe in a Paper contributed to this Society* that the latent heat is connected with osmotic pressure by the following nearly exact equation :-

$$
\mathrm{H}_{i} \dagger=s \mathrm{~T}^{2} \frac{\partial}{\partial \mathrm{T}}\left(\frac{\mathrm{P}}{\mathrm{T}}\right)
$$

Since for a perfect gas $\mathrm{P} / \mathrm{T}$ is independent of temperature, the latent heat of dilution would be zero if the osmotic pressure followed exactly that law. The value of $\mathrm{H}_{i}$ gives, therefore, a measure of the degree of departure from the law of perfect gases so far as change of temperature is concerned. Not many values of this quantity are yet known. For sugar there are a few determined by Stackelberg. Mr. Orson Wood, M.Sc., has recently determined some more at $20^{\circ} \mathrm{C}$. at my suggestion, and I am able to quote some previously unpublished results. The values obtained by him have been determined with very great care, and it is interesting to know that they lie on a curve passing through Stackelberg's points, so that both sets confirm one another. In the following table. I give these values for certain concentrations, together with the values of $y=\frac{\partial}{\partial \mathrm{T}}\left(\frac{\mathrm{P}}{\mathrm{T}}\right)$ calculated from them and from Morse's values at $10^{\circ} \mathrm{C}$. and $30^{\circ}$ by assuming $y$ to be uniform between those temperatures.

\begin{tabular}{|c|c|c|c|}
\hline $1,000 \frac{n}{M^{\circ}}$ & $\mathrm{H}_{i}$ cal./gram. & $y$ calculated from $\mathrm{H}_{i}$. & $\begin{array}{c}\text { Calculated from Morse's } \\
\text { Observations. }\end{array}$ \\
\hline $\begin{array}{r}\cdot 5 \\
\cdot 8 \\
\cdot 9 \\
\text { I.0 } \\
\text { I.5 } \\
2 \cdot 0 \\
4 \cdot 0\end{array}$ & $\begin{array}{ll}- & 04 \\
- & 07 \\
- & 09 \\
- & .11 \\
- & .245 \\
- & .415 \\
- & 1.12\end{array}$ & 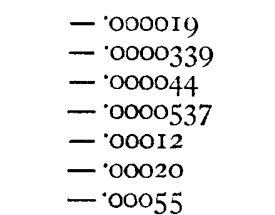 & 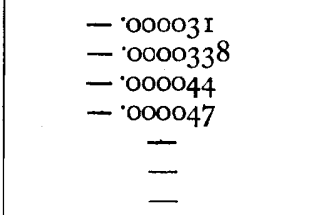 \\
\hline
\end{tabular}

The correspondence between the values of $y$ calculated in the two ways is not exact ; but the values are of the right order. It must not be thought, however, that the whole of Morse's determinations will stand the application of this test; it is far too searching. This can be seen even by a casual examination of an entire table of values of $P / T$ calculated from his numbers. Nor are these latent heats known at other temperatures. These are being obtained by Mr. D. O. Wood, and will constitute an important check upon determinations of osmotic pressure. In fact, in my opinion, values of osmotic pressures calculated from heats of dilution and from a standard set at some most convenient temperature (say $\circ^{\circ} \mathrm{C}$.) may be considerably more accurate than those determined directly.

Some light can be thrown on the possible physical meaning of the exist-

* Trans. Faraday Soc., vol. xi, part I, p. 22, I9I5.

$\dagger$ The value $\mathrm{H}_{i}$ is strictly the internal latent heat of dilution, but it is very nearly identical with the latent heat due to ordinary mixing. 


\section{THE KINETIC THEORY OF OSMOTIC PRESSURE}

ence of a latent heat by considering what the value of $T^{2} \frac{\partial}{\partial T}\left(\begin{array}{l}P \\ T\end{array}\right)$ is according to van der Waals' equation. If in the equation

$$
(\mathrm{P}+\mathrm{K})(v-b)=\mathrm{RT},
$$

$\mathrm{K}$ and $\mathrm{T}$ be assumed independent of temperature,

$$
T^{2} \frac{\partial}{\partial T}\left(\frac{P}{T}\right)=K
$$

and this would require $\mathrm{K}$ to be negative, which, in my opinion, is exceedingly unlikely to be the case, although the possibility of it has been entertained. However, it is now admitted that $\mathrm{K}$ is a function of temperature, and therefore

$$
\frac{\partial}{\partial T}\left(\frac{P}{T}\right)=-\frac{\partial}{\partial T}\left(\begin{array}{l}
K \\
\frac{T}{T}
\end{array}\right) ;
$$

or, if $\mathrm{P}$ increase less fast than the absolute temperature, $\mathrm{K}$ increases faster than it. This is not at all impossible.

According to the view which has been dealt with in this Paper in detail, it is $b$ that is a function of temperature; and $\mathrm{K}$ has been ignored. This gives

$$
\frac{\partial}{\partial \mathrm{T}}\left(\frac{\mathrm{P}}{\mathrm{T}}\right)=\frac{\mathrm{R}}{(v-b)^{2}} \frac{\partial b}{\partial \mathrm{T}^{\prime}}
$$

and the negative value of the left-hand side signifies that $b$ diminishes with temperature rise. My reason for ignoring $\mathrm{K}$ is that if it is taken into account $b$ must be even larger than the numbers I have given. It is difficult to discriminate between the above alternatives; all of them are equally consistent with the observed heats of dilution. In the case of dilute gases for which $b$ is small compared with $i$,

$$
p+\mathrm{K}=\frac{\mathrm{RT}}{v}\left(\mathrm{I}+\frac{b}{v}+\quad\right)
$$

and we may write $p=\mathrm{A}^{\mathrm{T}}+\mathrm{B}$ where the effects arising from $\mathrm{K}$ and $b$ are merged in the single term B. This indicates still further the difficulty of distinguishing between them.

\section{Solubility.}

The variation of the solubility of cane sugar with temperature can be accounted for in the main by assuming that it is due to the release of the water of hydration. The values of hydration numbers, for saturated solutions, that would be required if the variation were wholly due to this cause are given under the heading $a$ below :

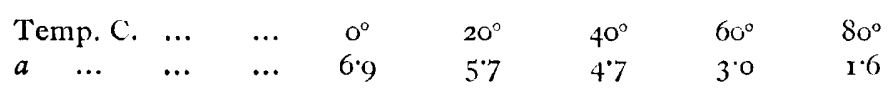

